\title{
Profil Microbiologique, Sensibilité Aux Antimicrobiens Et Résultat Du Traitement Des Abcès De Cornée Au CHU-IOTA
}

\author{
Microbiological Profile, Antimicrobial Susceptibility And Treatment Outcome Of Corneal \\ Abscesses At Iota Teaching Hospital
}

\author{
Elien G Y RR ${ }^{1 *}$,Bakayoko $S^{1}$,Simaga AS $^{1}$,Sissoko $M^{1}$,Nioumanta $M^{1}$,Sylla $A^{1}$,Diabaté \\ $\mathbf{R C N}^{\mathbf{1}}$, Traoré $\mathbf{L}^{\mathbf{1}}$.
}

1-Centre Hospitalier et Universitairedel'Institut de l'Ophtalmologie Tropicaled'Afrique (CHU-IOTA) de l'Université des Sciences, des Techniques et des Technologies de Bamako (USTTB), Bamako, Mali.

\section{* Correspondance : Dr Elien GYRR : CHU-IOTA, BP : 1428, Bamako-MALI courriel : rodrigueelien@yahoo.fr}

\section{Résumé}

Introduction : L'abcès de la cornée ou kératite infectieuse ou kératite suppurative se définit comme une infection de la cornée par un germe (bactérie, champignon, parasite) associée à des signes d'inflammation. Il s'agit d'une infiltration purulente du stroma cornéen. Il survient très rarementsur une cornée saine maisapparait plus fréquemmentsur un épithélium cornéen défectueux. L'abcès de la cornée est une urgence diagnostique et thérapeutique pouvant entrainer la perte anatomique et/ou fonctionnelle du globe oculaire; d'où la réalisation de cette étude dont le but est de contribuer à l'amélioration de la prise en charge de l'abcès de cornée à Bamako.

Méthode : il s'agissait d'une étude transversale, analytique et descriptive d'une durée de 16 mois allant de décembre 2019 à avril 2020.

Résultats : Durant notre étude, 286 prélèvements bactériologiques étaient revenus positifs sur le total de 472 prélèvements bactériologiques réalisés. Les germes majoritairement isolés étaient Staphylococcus aureus (48,11\%) suivi de Pseudomonas aeruginosa (15,19\%).l'abcès de cornée constitue l'une des principales causes de la morbidité oculaire et de cécité dans le monde et surtout dans les pays en voie de développement où l'hygiène est insuffisante.

Conclusion: Notre étude souligne l'importance de la prise en charge adaptée au cas par cas des abcès de cornée.

Mots clés : Abcès de cornée, germes, traitement, évolution, Bamako.

\begin{abstract}
Introduction: Corneal abscess or infectious keratitis or suppurative keratitis is defined as an infection of the cornea by a germ (bacterium, fungus and parasite) associated with signs of inflammation. It is a purulent infiltration of the corneal stroma. It occurs very rarely on a healthy cornea but appears more frequently on a defective corneal epithelium. Corneal abscess is a diagnostic and therapeutic emergency which can lead to anatomical and/or functional loss of the eyeball; hence the realization of this study whose aim is to contribute to the improvement of the management of corneal abscess in Bamako.
\end{abstract}

Method: this was a cross-sectionnal, analytical and descriptive study lasting 16 months from December 2019 to April 2020.

Results: During our study, 286 bacteriological samples came back positive out of a total of 472 bacteriological samples taken. The germs mainly isolated were Staphylococcus aureus $(48.11 \%)$ followed by Pseudomonas aeruginosa (15.19\%).Corneal abscess is one of the main causes of ocular morbidity and blindness in the world and especially in developing countries where hygiene is inadequate.

Conclusion: Our study underlines the importance of case-by-case management of corneal abscesses.

Key words: Corneal abscess, germs, treatment, evolution, Bamako. 


\section{Introduction}

Les kératites infectieuses sont l'une des importantes causes de la morbidité oculaire dans le monde[1]. Une mauvaise ou insuffisante prise en charge des kératites infectieuses aboutit inéluctablement à la perte fonctionnelle et/ou anatomique du globe oculaire [1-2].Son incidence est de 113 pour 100 000 personnes par an en Asie [3]. À Madagascar, la fréquence hospitalière des kératites infectieuses était de 9,96\% [4].En Afrique de l'Ouest, plusieurs études ont été menées sur la kératite en général [57].Par contre les analyses microbiologiques suivies des tests de sensibilité aux antimicrobiens au cours des kératites infectieuses étaient insuffisamment réalisés en Afrique en général, notamment à Bamako. Ce qui justifie la réalisation de cette étude dont l'objectif était de contribuer à la description du profil microbiologique et de la sensibilité aux antimicrobiens des kératites infectieuses afin de proposer un schéma thérapeutique de première intention.

\section{Patients Et Méthodes}

Nous avons conduit une étude transversale, analytique et descriptive au sein du service de l'hospitalisation du CHU-IOTA (Bamako, Mali) des patients hospitalisés pour abcès cornéen grave. La durée de notre étude est 16 mois, allant du 1er Décembre 2018 au 30 Avril 2020.Nous avons inclus par échantillonnage non probabiliste dans l'étude 236 patients atteints d'abcès de cornée.Tous les patients ont bénéficié d'un examen ophtalmologique complet à la lampe à fente, d'un prélèvement conjonctival et d'un prélèvement cornéen. Les prélèvements conjonctival et cornéen ont été adressés au laboratoire pour des recherches bactériologique, mycologique et parasitologique. Ces prélèvements ont été pratiqués le jour de l'admission chez les patients par le médecin ophtalmologiste membre de l'équipe de l'étude avant tout traitement anti infectieux. Nous avons effectué pour le prélèvement cornéen, un grattage cornéen profond des berges et du fond de la lésion sous une loupe après instillation d'un collyre anesthésique, à l'aide du biseau de l'aiguille de 21 Gauge à embout vert ou d'un écouvillon stérile humidifié. Le prélèvement conjonctival était obtenu par écouvillonnage. Le produit de grattage cornéen est étalé puis fixé sur lame puis coloré au GRAM, May-GrünwaldGiemsa ou Grocott pour l'examen direct. Ensuite les prélèvements ont été mis en culture sur gélose au sang, gélose chocolat, gélose Sabouraud et dextrose (SDA) et enfin bouillon Cour-Cervelle (BCC). Les bactéries et champignons cultivés ont été identifiés selon le protocole standard. Pour l'étude de la sensibilité des bactéries aux antibiotiques nous avons recouru à la méthode Kirby-Bauer de diffusion sur gélose. Cette méthode permet la lecture directe de la sensibilité des germes en les classant selon la relation entre CMI et diamètre de l'inhibition autour du disque d'antibiotique. Le diamètre d'inhibition est comparé à des diamètres critiques définis par le Comité de l'Antibiogramme de la Société Française de Microbiologie. Si le diamètre d'inhibition est en dessous du diamètre critique inférieur la souche est classée Résistante à l'antibiotique,au-dessus du diamètre critique supérieur la bactérie est classée Sensible, et Intermédiaire si son diamètre d'inhibition est compris entre les valeurs inférieure et supérieure du diamètre critique.Dès que le diagnostic clinique était retenu et les prélèvements microbiologiques réalisés, un traitement empirique était instauré. Ce traitement empirique initial consistait en une association synergique active sur les bactéries Gram+ et Gram-, aux posologies efficaces et non toxiques pour la cornée. Puis,il a été ensuite adapté aux résultats de l'analyse microbiologique. Ce traitement concernait les voies topique et parentérale. Notre étude était autorisée par les autorités académiques et administratives du CHU-IOTA. Elle était réalisée dans le respect des recommandations de la déclaration d'éthique d'Helsinki au sujet des études sur les êtres vivants.Les données ont été recueillies à l'aide d'un questionnaire préétabli, puis saisies et analysées au moyen du logiciel EPI info 7.0 du CDC Atlanta. Le traitement de texte a été fait avec le logiciel Microsoft Word 2007 et le logiciel Microsoft Excel 2007 a été utilisé pour la production de graphiques et de tableaux. Le test du Chi 2 a été utilisé avec un seuil de sensibilité de $5 \%$. Les variables étudiées étaient les suivantes :

$\checkmark$ Variables épidémiologiques (âge, sexe; profession, antécédents pathologiques, délai de consultation)

$\checkmark \quad$ Variables cliniques : signes fonctionnels (baisse de l'acuité visuelle, douleur, écoulement des larmes, photophobie) ; signes physiques (hyperhémie conjonctivale, infiltration cornéenne purulente, ulcération cornéenne, œdème cornéen, hypopion) ; le site et le type du prélèvement microbiologique.

$\checkmark$ Variables microbiologiques: coloration, examen direct, culture, sensibilité des germes aux antimicrobiens.

\section{Résultats}

Durant notre étude nous avons réalisé 472 prélèvements bactériologiques, répartis en 236 prélèvements cornéens et 236 prélèvements de la conjonctive.Les prélèvements positifs étaient au nombre 79 , soit un taux de positivité de $16,73 \%$ 


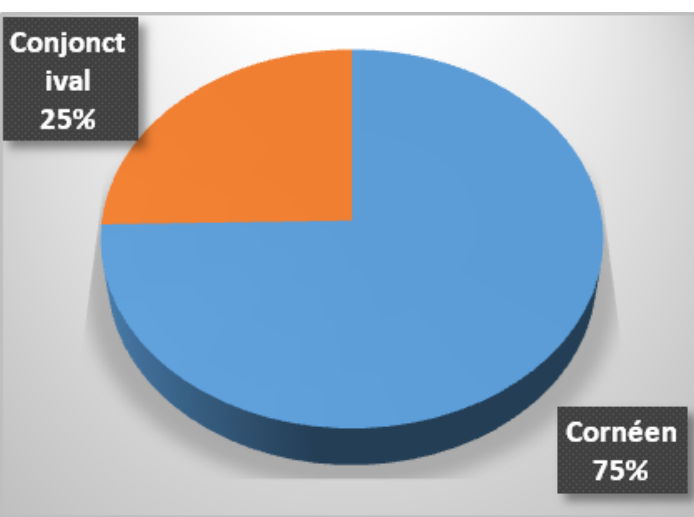

Figure 1 : Répartition des prélèvements positifs selon le site du prélèvement.

La presque totalité des prélèvements positifs étaient issus de la cornée.

Tableau I : Répartition des germes isolés en fonction des facteurs de risque

\begin{tabular}{|c|c|c|c|c|}
\hline FR & \multicolumn{2}{|c|}{ Germes isolés } & \multirow{2}{*}{$\begin{array}{l}\mathbf{N} \\
25\end{array}$} & \multirow{2}{*}{$\begin{array}{c}\% \\
31,7\end{array}$} \\
\hline \multirow{5}{*}{ 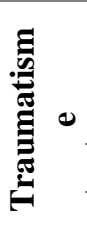 } & BGP & S.aurus & & \\
\hline & & S.epidermidis & 4 & 5,7 \\
\hline & & S.peumoniae & 10 & 12,7 \\
\hline & $\mathrm{BGN}$ & Pseudomonasspp & 12 & 15,2 \\
\hline & Myc & Fusariumspp & 1 & 1,3 \\
\hline \multirow{4}{*}{ 莺 } & BGP & S.aurus & 13 & 16,5 \\
\hline & & S.epidermidis & 1 & 1,27 \\
\hline & BGN & Moraxellaspp & 1 & 1,27 \\
\hline & Myc & Fusariumspp & 1 & 1,3 \\
\hline \multirow{3}{*}{ 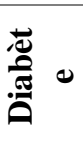 } & BGP & S.pneumoniae & 4 & 5,1 \\
\hline & BGN & Pseudomonas spp & 1 & 1,3 \\
\hline & Myc & Fusariumspp & 2 & 2,5 \\
\hline \multirow{4}{*}{ 丑 } & BGP & S.pneumoniae & 1 & 1,3 \\
\hline & & $\begin{array}{l}\text { Corynebacteriums } \\
\text { pp }\end{array}$ & 2 & 2,5 \\
\hline & BGN & H.influenzae & 1 & 1,3 \\
\hline & & otal & 79 & 100 \\
\hline
\end{tabular}

La majorité des germes isolés était des bactéries GRAM + $(75,96 \%)$, suivie des bactéries GRAM $(18,97 \%)$ )et des champignons $(5,07 \%)$.

Chez les patients immunocompétents, les bactéries GRAM + les plus fréquents étaientStaphylococcus aurus (48,11\%). Et Pseudomonas aeruginosa $(15,19 \%)$ était le plus représenté des bactéries GRAM -.

D'autres germes rares, en plus du Streptococcus pneumoniae le plus fréquent $(6,33 \%)$, ont été isolés chez les patients immunodéprimés :

Corynebacteriumpseudodiphtheriticum (2,53\%) et Haemophilus influenzae (1,27\%).

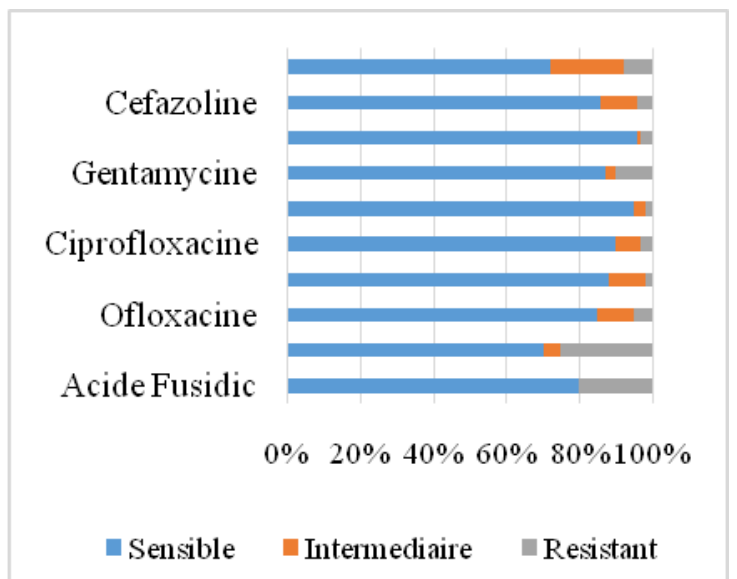

Figure 2 : Répartition des antibiotiques selon la sensibilité des bactéries Gram+

Les familles d'antibiotique sensibles sur les bactéries GRAM + de façon de décroissante étaient: les Phénicolés (Chloramphénicole), les Quinolones du groupe des Fluoroquinolones (Ciprofloxacine, Norfloxacine, Ofloxacine), les Céphalosporines de $1^{\text {ère }}$ génération (Cefazoline), l'acide fusidique, les Polypeptides (Bacitracine et Neomycine), les Aminoglycosides (Gentamycine et Trobamycine).

Figure 3 : Répartition des antibiotiques selon la sensibilité des bactéries GRAM -

Les familles d'antibiotique sensibles sur les bactéries GRAM - de façondécroissante étaient : les Quinolones du groupe des Fluoroquinolones (Ciprofloxacine, Norfloxacine, Ofloxacine), les Aminoglycosides (Gentamycine et Trobamycine), les Polypeptides (Bacitracine et Neomycine), les Phénicolés (Chloramphénicole), les Céphalosporines de $1^{\text {ère }}$ génération (Cefazoline), l'acide fusidique.

Les abcès de cornée à fusariumspp ont été traités avec l'amphotéricine B collyre.

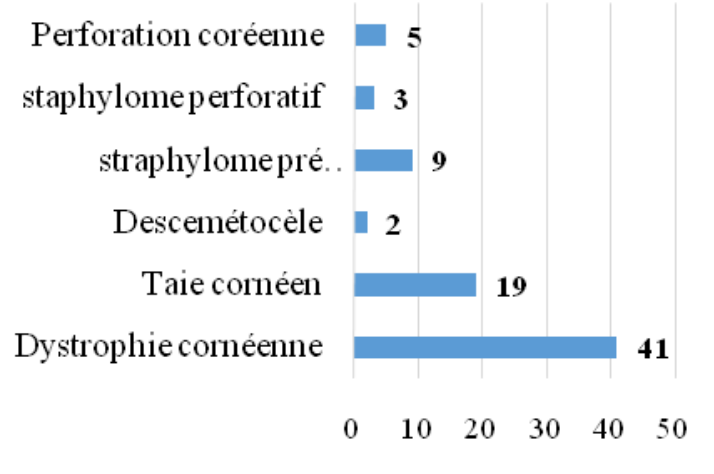

Figure 4 : Répartition des patients selon l'évolution après traitement.

L'évolution sous traitement bien conduit a abouti à la l'énucléation de l'œil de 10,12\% patients, $13,92 \%$ des patients sont à risque de rupture du globe oculaire et 75,94\% des patients avaient des 
anomalies de la fonction visuelle à des degrés variables.

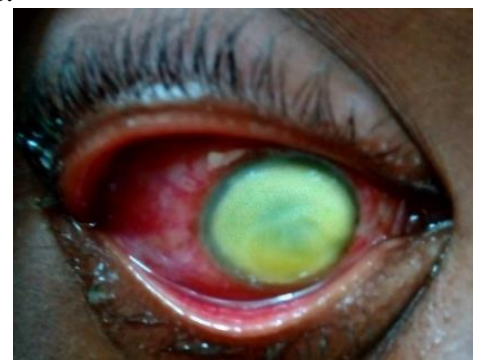

A-Abcès total de la cornée.

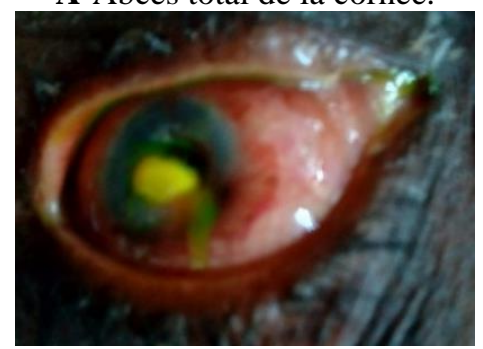

B-Fonte purulente de la cornée

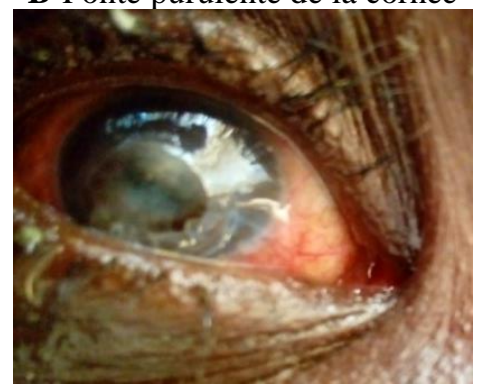

C-Staphylome cornéen perforatif.

Figure 5 :

\section{Discussion}

Dans notre étude, le taux de positivité des prélèvements microbiologiques était de 16,73\%. Ce résultat est comparable à celui trouvé à Dakarpar Seck et al[7] mais inférieur au résultat retrouvé par Limaiem et al dans une étude similaire réalisée au Maroc [8], qui ont respectivement trouvé $17 \%$ et $42 \%$ de taux de possitivité. L'utilisation des médicaments oculaires avant l'admission, le delai écoulé entre l'apparition des symptômes et la consultation à l'hôpital, le diamètre du défect épithélial ou de l'infiltrat dans l'œil infecté, sans oublier la mauvaise qualité du frottis prélevé sur la cornée ou sur la conjonctive dans certains cas justifieraient le faible taux de positivité observé dans notre serie. Cependant, 3/4 de nos cultures positives provenaient du grattage cornéen. Ce résultat est similiaire aux taux de positivité rapportés par la littérature selon laquelle, le taux de positivité des cultures cornéennes oscille entre 60 et $84 \%$ [9-11]. Comme le corrobore la littérature au sujet de la culture des prélèvements oculaires comme gold standard pour le diagnostic microbiologique des kératites infectieuses. En outre, nous avons observé que les abcès bactériens aient été les plus fréquents $(94,93 \%)$, avec une prédominance des bactéries Gram+ (75,96\%). Notre observation se rapprochait de celles retrouvées dans la sous région[7,8,11,12]. Il en est de même des germes fréquements isolés, à savoir le staphylocoque doré $(37 \%)$ et le pseudomonas aeruginosa (27 \%). Ces observations sont comparables aux données de la littérature [7-13]qui stipule que l'incidence des germes variait selon la localisation géographique, et le climat.Ainsi, dans les régions chaudes et humides, les abcès fongiques et à pseudomonas seraient plus fréquents alors que la prévalence des abcès à staphylocoque et à streptocoque augmenterait dans les régions a climat froid. Par ailleurs, l'identification des germes est souvent dificile en raison de la délicatesse des prélèvements oculaires offrant une faible quantité d'échantillon à prélèver et de la contamination possible des prélèvements par La flore bactérienne commensale de la conjonctive et des paupières. De ce fait, un germe ne peut être considéré comme pathogène, que si sa culture ait été monomicrobienne, qu'il y ait une concordance entre les données de l'examen direct et la culture. Ainsi, pour une bonne analyse microbiologique nous optons préférentiellement pour les cultures au détriment des frotttis. Dans notre étude, les bactéries Gram + étaient plus sensibles aux antibiotiques de la famille des phéicolés, des quinolones (groupe fluoroquinolone notament), les céphalosporines de la $1^{\text {ère }}$ génération et l'acide fusidique. Parcontre les bactéries Gram - étaient sensibles aux quinolones, aux aminoglycosides et aux polypeptides. Nos données se rapprochent de la littérature et se justifier par le spectre d'activité des différentes familles des antibiotiques sus mentionnées [717].Fort de ces observations, il nous semble pertinent de proposer en premiere intension l'association synegique de fluoroquinolone et de l'acide fusidique. Considérant la toxicité cornéenne des fluoroquinolones au délà du $4^{\mathrm{e}}$ jour d'utilisation [18-20], nous sugerons son remplacement au 4 e jour par un phénicolé ou un polypeptide pour la suite du traitement. Enfin, l'évolution des patients dans notre étude aboutisait soit à la perte fonctionelle du globe oculaire à des dégrés variables soit à la perte anatomique et fonctionnelle du globe oculaire. Ceci étaiet problablement dû au retard d'admission des patients, qui se présentaient généralement à l'hôpital soit avec une altération sévère de la cornée, soit à un stade tardif de l'abcès. Alors que, la prise en charge initiale influe grandement au facteur pronostic et conditionne l'évolution du patient. Le rythme d'administration des collyres antibiotiques influence également l'évolution rapide ou lente des abcès cornéens, avec une dose de charge et une dose d'entretien.

\section{Conclusion}

La kératite infection est une affection potentiellement cécitante. Il s'agit d'une urgence diagnostique et therapeutique. Sa prise en charge optimale nécessite l'identification de l'agent 
pathogène et sa sensibilité aux antimicrobiens usuels. Notre étude a montré une prédominance des bactéries Gram + notamment le genre Staphylococcus. Les antibiotiques de la famille des Quinolones (particulièrement les Fluoroquinonlones) étaient sensibles aussi bien pour les bactéries Gram + que les bactéries Gram -. La perte fonctionnelle ou la perte anatomique du globe était l'issue commune de nos patients. Nous insistons sur la coopération étroite entre l'ophtalmologiste, l'infectiologue et le biologiste pour une meilleure approche thérapeutique des abcès de cornée dans nos hôpitaux. Nous espérons que nos résultats serviront de base aux professionnels de la santé oculaire pour le traitement et la surveillance des kératites inectieuses dans la pratique courante.

Conflit d'interet : Aucun

\section{Références}

1. Baklouti K, Ayachi M, Mhiri N, Mrabet A, Ben $\mathbf{N}$, Ben R. Les abcès cornéens présumés d'origine bactérienne Bull Soc Belge Ophtalmol 2007 ; 305 : 39-44

2. Burillon C.Douleur et pathologie cornéennes.J Fr Ophtalmol,2000,23:386.

3. Nithya $\mathbf{V}$, Bhaskar A.A review on microbial keratitis.World J Pharm Res, 2014,3(7) :189-201.

4. Raobela L, Ralibert JN, Andriatsilavo RR, Randrianarisoa HL, Volamarin RF, Bernardin PL.Les abcès cornéens graves présumés d'origine bactériennes vus au Centre hospitalier et Universitaire d'Antanarivo. Rev.anesth.réanim.med.urgence $2015 ; 7(1): 10-14$.

5. Agbodza KMD.Etude epidemiologique des kératites à l'IOTA.thèse de Doctorat de Médecine.2008.FMOS-USTTB.85p

6. Amadou B. Aspects épidemiologique des Kératites au Centre de Santé de Référence de la commune V $\mathrm{du}$ District de Bamako. thèse de Doctorat de Médecine.2014.FMOS-USTTB.97p

7. Seck SM, Diakhaté M, Oulfath A, Sow MN, Dieng M, Gueye NN.Severe Infectious Keratitis in Tropical Environments: 118 Cases Collected Over 10 Years.Med Sante Trop. 2019, 1;29(2):151-154.

8. Limaiem R, Mghaieth F, Merdassi A, Mghaieth K, Aissaoui A, El Matri L.Les abcès graves de la cornée : à propos de 100 cas. J Fr Ophtalmol. 2007, 30 (4) : 374-379.
9. Vajpayee RB, Dada T, Saxena R, Vajpayee M, Taylor HR, Venkatesh P, Sharma N. Study of the first contact management profile of cases of infectious keratitis: a hospital-based study. Cornea, 2000; 19:52-6.

10. Gopinathan U, Sharma S, Garg P, Rao GN. Review of epidemiological features, microbiological diagnosis and treatment outcome of microbial keratitis: Experience of over a decade. Indian J Ophthalmol.2009; 57(4):273-9.

11. Oladigbolu K, Rafindadi A, Abah E, Samaila E. Corneal ulcers in a tertiary hospital in Northern Nigeria. Ann Afr Med. 2013; 12(3).

12. Raobela L, Ralibert JN, Rajaona RA, Randrianarisoa HL, Volamarina RF, Bernardin PL. Les abcès cornéens graves présumés d'origine bactérienne vus au Centre Hospitalier Universitaire d'Antananarivo. Revanesth-RéanimMedUrg. 2015;7(1):10-4.

13. Kumar A, Pandya S, Kavathia G, Antala S, Madan M, Javdekar T. Microbial keratitis in Gujarat, Western India: findings from 200 cases. Pan Afr Med J. 2011; 10:48.

14. Daniell M, Mills R, Morlet N. Microbial keratitis: what's the preferred initial therapy? $\mathrm{Br} J$ Ophthalmol, 2003;87:1167-9.

15. Mallari PLT, McCarty DJ, Daniell M, Taylor H.Inceased incidence of corneal perforation after topical Fluoroquinolone treatment for microbial keratitis. Am J Ophthalmol, 2001;131:131-3.

16. Fong CF, Tseng CH, Hu FR, Wang IJ, Chen WL, Hou YC. Clinical characteristics of microbial keratitis in a university hospital inTaiwan. Am J Ophthalmol, 2004; 137:329-36.

17. Wang N, Yang $\mathbf{Q}$, Tan $Y$, Lin $\mathbf{L}$, Huang $\mathbf{Q}$, Wu K. Bacterial Spectrum and Antibiotic Resistance Patterns of Ocular Infection: Differences between Externaland Intraocular Diseases. J Ophthalmol.2015; 813979:7p.

18. Moreira LB, Lee RF, de Oliveira C, Labree $L$, McDonnell PJ. Effect of topical fluoroquinolones on corneal re-epithelialization after excimer laser keratectomy. J CataractRefractSurg, 1997;23:8458.

19. Reviglio VE, Hakim MA, Song JK, O'Brien TP. Effect of topical fluoroquinolones on the expression of matrix metalloproteinases in the cornea. $B M C$ Ophthalmol, 2003;3:10.

Walter K, Tyler ME. Severe corneal toxicity after topical fluoroquinolone therapy: report of two cases. Cornea, 2006;25:855-7. 